FORMATION Formation emploi

Revue française de sciences sociales

126 | Avril-Juin 2014

Qu'apprend-on des expérimentations sociales?

\title{
La sécurisation des parcours des apprentis à l'épreuve du choix politique
}

The bolstering up of the apprentice pathways in the light of policy Absicherung des beruflichen Werdegangs von Auszubildenden vor dem Hintergrund politischer Entscheidungen

La protección de los itinerarios de los aprendices sometida a la decisión política

Jean Bourdon, Christine Guégnard et Claire Michot

\section{OpenEdition}

Journals

Édition électronique

URL : http://journals.openedition.org/formationemploi/4196

DOI : 10.4000/formationemploi.4196

ISSN : 2107-0946

Éditeur

La Documentation française

Édition imprimée

Date de publication : 10 juillet 2014

Pagination : $79-98$

ISSN : 0759-6340

Référence électronique

Jean Bourdon, Christine Guégnard et Claire Michot, «La sécurisation des parcours des apprentis à l'épreuve du choix politique », Formation emploi [En ligne], 126 | Avril-Juin 2014, mis en ligne le 18 août 2014, consulté le 30 octobre 2020. URL : http://journals.openedition.org/formationemploi/4196 ; DOI : https://doi.org/10.4000/formationemploi.4196 


\title{
La sécurisation des parcours des apprentis à l'épreuve du choix politique
}

\author{
JEAN BOURDON \\ Directeur de recherche CNRS en économie, à l'Institut de recherche sur l'éducation, université de \\ Bourgogne \\ Christine GuÉGNARD \\ Chargée d'études à l'Institut de recherche sur l'éducation, université de Bourgogne, pour le centre \\ associé au Céreq, Dijon, sciences de l'éducation \\ Claire Michot \\ Chargée d'études à l'université de Bourgogne, sciences de l'éducation
}

Résumé

La sécurisation des parcours des apprentis à l'épreuve du choix politique

L'histoire originale d'une expérimentation sur la sécurisation des parcours des apprentis, à l'épreuve d'une décision politique régionale, fournit l'opportunité d'étudier la nature des relations et le type de participation ou de transaction à l'œuvre. La propension des acteurs, aux stratégies différenciées et porteurs d'informations spécifiques, à défendre leur point de vue institutionnel, les alliances et les compromis qui se construisent pour le choix d'une coopération pérenne dans un avenir incertain, caractérisent la gouvernance territoriale en matière de formation professionnelle.

Mots clés : expérimentation sociale, politique régionale de la formation professionnelle, abandon des études, apprenti, formation en alternance

Abstract

The bolstering up of the apprentice pathways in the light of policy

The original situation of an experiment on the security of the apprentice pathways in the light of regional policy, provides an opportunity for studying the nature of the relationships and the form of participation or transaction that takes place. The territorial governance of vocational training is characterized by the propensity for the actors (with the different strategies and possessing specific information) to defend their own institutional point of view, and by the alliances or the compromises made in choosing long term co-operation in an uncertain future.

Keywords : social experiment, regional cvt policy, drop out, apprentice, sandwich training Journal of Economic Literature: H 52, I 21

Traduction : Auteurs 
La loi du 7 janvier 1983 confere aux régions françaises une compétence de droit commun pour la mise en place d'actions dans le domaine de l'apprentissage et de la formation professionnelle continue. Dès lors, la décentralisation a déplacé les lieux de décision, modifié le cadre des analyses, multiplié les acteurs et transformé leur rôle (Giffard, Guégnard, 2003). Ainsi, le Conseil régional assure la gouvernance du territoire avec une pluralité d'acteurs publics ou privés, institutionnels ou associatifs, avec des principes de transparence et d'arbitrage associés à cette démarche. Son rôle s'inscrit dans un univers d'interdépendance en mobilisant l'ensemble des agents concernés; il s'agit d'impulser une politique de formation professionnelle, vectrice d'insertion sociale et professionnelle.

Dans ce cadre, l'apprentissage occupe une place particulière. Cette voie de formation par alternance est traditionnellement présente en Bourgogne, où la part des apprentis avoisine les $6 \%$ de la population totale des 16-25 ans. Au fil du temps, le nombre d'apprentis a progressé pour atteindre, en 2007, un palier de 12000 jeunes, mais il décline depuis 2009. Cette situation est le reflet du tissu économique, de la capacité des entreprises à accueillir des apprentis, de la demande des jeunes, de l'offre de formation en CFA (centres de formation des apprentis). Ce développement résulte aussi des politiques du conseil régional conduites en partenariat avec les représentants des services de l'État et les acteurs économiques et sociaux, qui définissent les lignes directrices et leurs actions en matière d'apprentissage. Ainsi, le contrat d'objectifs et de moyens ${ }^{1}$ engage l'État et la région de Bourgogne à investir plus de onze millions d'euros par an pour le développement de l'apprentissage.

Améliorer la qualité de l'apprentissage est un objectif majeur actuel en région. Certes, l'apprentissage offre aux jeunes la possibilité d'acquérir une expérience, une qualification, un diplôme et, parfois, une embauche dans l'entreprise formatrice'. Toutefois, ces avantages ne doivent pas masquer la réalité de ce mode de formation (Moreau, 2003) et les difficultés des jeunes qui peuvent entraîner une rupture du contrat, un abandon de la formation, voire un décrochage ( $c f$. encadré $\mathbf{1}$ " Méthodologie »). Selon diverses études, près d'un contrat d'apprentissage sur quatre est rompu avant son terme $e^{3}$.

Les motifs de rupture sont variés, souvent liés à des problèmes de mésententes entre apprenti et maître d'apprentissage ou employeur, des erreurs d'orientation ou de choix du métier, ou encore à des conditions de travail difficiles ou de mauvaise qualité, mais aussi aux situations personnelles de l'apprenti ou aux difficultés économiques de l'entreprise. La résiliation est un phénomène difficile à appréhender, car il n'existe pas à l'heure actuelle de suivi régulier des contrats au plan national. Les données sont éparses, souvent partielles, variant selon les territoires et les métiers. Dans cette incertitude, une constante : ces rup-

1. Institué par la loi du 18 janvier 2005 pour la programmation de la cohésion sociale.

2. Cf. Sollogoub, Ulrich, 1999 ; Abriac et alii, 2009 ; Céreq, 2011 ; Alet, Bonnal, 2013.

3. Les chiffres vont de $25 \%$ à $27 \%$ au plan national, à près de $30 \%$ dans le Nord-Pas-de-Calais ou en Bretagne (Dep, 1995, 1997 ; Cart et alii, 2007 ; Chambres de commerce, 2010 ; Bentabet et alii, 2012 ; GREF, 2013). 
tures n'interviennent pas seulement lors de la période d'essai de deux mois mais tout au long du cursus des jeunes. Un autre résultat invariable est que l'hôtellerie-restauration est signalée comme le secteur spécifique des résiliations.

Face à cette situation, plusieurs expériences ont été réalisées afin de sécuriser l'orientation des jeunes vers l'alternance et réduire les ruptures ou le décrochage. Le Fonds d'expérimentation pour la jeunesse a, d'une part, soutenu des initiatives innovantes destinées à favoriser la réussite des jeunes; d'autre part, il a financé des évaluations externes qui devaient guider les réflexions locales et nationales sur de possibles généralisations de ces dispositifs à d'autres territoires. C'est dans ce contexte que nous nous proposons d'étudier l'histoire particulière d'une expérimentation visant à prévenir les ruptures des contrats d'apprentissage : quels sont les effets d'une action de tutorat menée auprès des apprentis et des employeurs par les trois missions locales de Côte-d'Or ? Quelle est la nature de la coopération instituée entre agents ? Quelles sont les conclusions de l'évaluation quant au transfert du dispositif vers d'autres zones de Bourgogne ou d'autres régions et enfin quelle est la décision prise par le conseil régional de Bourgogne quant à son extension ?

Le caractère pluriel des acteurs et des stratégies et la nature complexe du choix d'une politique dans un cadre de négociations et d'interactions sont mis en lumière. Nous proposons d'esquisser une analyse de la gouvernance d'un territoire en référence à la théorie des coûts de transaction de Williamson (1994, p. 349) : « En fait, toute relation, économique ou autre, qui prend la forme d'un problème contractuel (ou qui peut être décrit comme tel) peut être évaluée avantageusement selon les termes de l'économie des coûts de transaction. "L'auteur estime que certaines activités sont coordonnées par d'autres mécanismes que celui des prix, et que la relation contractuelle se substitue au marché. Pour réaliser ces transactions, les agents économiques peuvent rechercher des arrangements institutionnels alternatifs permettant de minimiser ces coûts. Lors de la mise en ouvre de ces transactions, les agents vont chercher à établir un contrat afin de fonder une coopération pérenne dans un avenir incertain. Le caractère particulier des relations qui se nouent entre les parties devient alors déterminant : l'existence d'une relation ni anonyme ni limitée dans le temps crée une dépendance qui modifie la règle contractuelle habituelle. L'analyse selon cette théorie s'intéressera donc aux relations entre les parties prenantes à la transaction, à travers l'histoire de cette expérimentation.

La première partie de l'article fournit les principales clés contextuelles afin de situer l'expérience nouvelle du tutorat dès le début du contrat d'apprentissage et l'implication d'un réseau d'acteurs. Elle se prolonge par un éclairage sur les caractéristiques des jeunes apprentis et les impacts du tutorat, après deux années d'observation. Le coeur de la troisième partie concerne les propositions quant aux pratiques transférables du dispositif, les temps d'appropriation et de négociation par les partenaires régionaux pour étendre la portée du dispositif, puis la décision finale du conseil régional. La nécessité de coordonner une multiplicité d'acteurs apparaît tant dans la mise en place du tutorat que dans le mode de gouvernance résultant de la décentralisation. Le rôle du conseil régional est souligné 
comme intermédiaire et acteur pivot en ce qui concerne l'affirmation de la fonction de coordination et de régulation dans l'espace régional (Comité de Coordination, 2000). Il doit coopérer avec l'ensemble des acteurs ou, autrement dit, ne pas gouverner mais assurer la gouvernance du territoire entre les acteurs.

\section{Un projet innovant pour sécuriser les parcours des apprentis}

L'apprentissage, mode de formation par alternance, repose sur un contrat de travail de type particulier, signé entre un jeune et un employeur. La découverte de l'entreprise, le suivi des cours dans un nouveau centre de formation, l'organisation du transport et du logement, modifient les repères et la vie des jeunes qui sortent du système scolaire. Si de nombreuses actions sont mises en place pour assurer un meilleur accompagnement des jeunes à l'entrée au lycée ou en première année de l'enseignement supérieur, elles sont plus rares pour les apprentis. Or, pour ces derniers, l'arrivée dans le monde de l'entreprise rend la transition encore plus ardue. Comme l'explique Moreau (2008 p. 128), "cette confrontation au travail, à l'entreprise, à ses règles et ses abus, est le point de rencontre de systèmes de valeurs-culture juvénile, culture d'entreprise, culture du métier-qui peinent parfois à trouver un équilibre". Les interventions existantes sont morcelées et reposent actuellement sur le suivi pédagogique des centres de formation, sur la fonction des chambres consulaires ou sur le suivi des missions locales pour les jeunes qu'elles ont orientés. Elles sont parfois tardives, davantage palliatives que préventives, et n'impliquent pas de réelle coordination des acteurs locaux.

Un projet innovant a été testé pour sécuriser les parcours des apprentis, porté par la mission locale de Beaune, financé par le Fonds d'expérimentation pour la jeunesse ${ }^{4}$ et évalué par l'Institut de recherche sur l'éducation (IREDU) : un accompagnement des apprentis et des employeurs dès la signature du contrat, sous forme d'un tutorat assuré par un conseiller de la mission locale, un acteur extérieur ne dépendant ni de l'entreprise ni du centre de formation. Ce dispositif instaure un accompagnement centré sur la relation jeune-employeur et une politique de prévention, avec la possibilité de transitions sécurisées par changement d'entreprise ou d'orientation. De plus, il contribue à la lutte contre le décrochage scolaire des jeunes, enjeu national des politiques éducatives menées en faveur de la jeunesse.

Avant d'exposer les principaux résultats de l'expérimentation, nous allons décrire, dans un premier temps, le processus et les modalités d'accompagnement mis en place auprès des jeunes et des employeurs de Côte-d'Or. Ensuite, les principaux acteurs engagés dans cette

4. Cette action a été financée aussi par le conseil régional de Bourgogne, le Fonds social européen, et la Direction régionale des entreprises, de la concurrence, de la consommation, du travail et de l'emploi (Direccte) de Bourgogne. 
action seront présentés, au regard des témoignages des personnels des missions locales qui, au cœur de cette expérience, révèlent les partenariats, les types de coopération mais aussi les contraintes.

\subsection{Un processus d'accompagnement}

L'expérience a été menée par les trois missions locales de Côte-d'Or auprès des apprentis et des entreprises de l'hôtellerie-restauration pour la première année du projet, auxquels s'ajoutent ceux du commerce et de la viticulture pour la seconde phase. Tous les acteurs (chambres consulaires, centres de formation, service académique de l'inspection de l'apprentissage, branche professionnelle) ont participé activement à l'amélioration de la prévention des ruptures.

Le processus d'accompagnement était conçu sur le modèle suivant : suite à l'envoi du contrat d'apprentissage par la chambre consulaire, la mission locale adressait un courrier d'information $^{5}$ au jeune et à l'employeur sur la désignation d'un tuteur externe. Celui-ci organisait ensuite une visite en entreprise pour rencontrer individuellement, puis conjointement, le jeune et son maître d'apprentissage, afin de présenter le dispositif, établir un premier diagnostic et instaurer un climat de confiance. Le tutorat impliquait un suivi par entretien mensuel de l'apprenti ou de l'employeur, en lien avec le centre de formation, et des interventions de tous types (logement, mobilité, souci financier, démotivation, conditions de travail, assiduité aux cours...). En cas d'alertes ou de difficultés signalées, un appui social ou une conciliation étaient mis en œuvre avec un bilan partagé, un plan d'amélioration défini et une vérification du bon déroulement après deux semaines dans l'entreprise. Puis, si la poursuite du contrat s'avérait impossible, la recherche d'une nouvelle entreprise dans le même secteur ou l'élaboration d'un nouveau projet professionnel était proposée. Ce schéma correspond bien à la nécessité, décrite par Steedman (2012), d'une action publique autour de l'apprentissage qui vise à réguler, par des transactions adéquates, les situations où les risques de marché sont importants.

Dès le début de l'expérimentation, le principe retenu était de présenter cet accompagnement de manière aléatoire à une partie du public cible, l'autre devant être informée seulement par lettre de la possibilité de faire appel au tutorat en cas de problèmes. Cette population constituait dès lors le groupe témoin. Mais par souci d'éthique, il avait été décidé, en comité de pilotage, qu’un jeune informé par lettre bénéficierait du tutorat s’il était signalé en difficulté ou en rupture de contrat ${ }^{6}$. De plus, au cours de l'évaluation, il

5. Pour l'hôtellerie-restauration, la lettre était signée par l'Union des métiers et des industries de l'hôtellerie (Umih), ce qui révélait son implication vis-à-vis des entreprises.

6. Au cours des deux années d'observation, 55 jeunes ont basculé du groupe information par lettre vers le groupe tutorat : 19 sont restés dans l'entreprise, 36 ont connu une rupture de contrat dont 13 ont quitté l'apprentissage. Pour ne pas interférer avec l'assignation aléatoire, ils ont été exclus des modélisations présentées dans cet article, ainsi que les 193 apprentis non bénéficiaires de l'expérimentation. 
est apparu que des jeunes inscrits dans les CFA n'étaient pas connus des missions locales et qu'on ne leur avait proposé ni lettre ni tutorat. Ces 193 jeunes ont été suivis et ont constitué un groupe de non-bénéficiaires.

\section{Encadré 1 : méthodologie}

Cet article repose en premier lieu sur les résultats d'une expérimentation menée en faveur des apprentis et des employeurs. L'IREDU (Institut de recherche sur l'éducation) a eu pour mission d'analyser les différences de parcours entre les apprentis bénéficiant du tutorat et les non-bénéficiaires. En particulier, nous nous intéressons, dans cet article, à la rupture du contrat d'apprentissage (ou la résiliation, dénomination juridique) et au décrochage. La rupture est définie ici comme une interruption du contrat avant le terme initialement prévu, que ce soit pendant ou après la période d'essai de deux mois, avec ou sans signature d'un autre contrat d'apprentissage (rupture à l'état brut). Le décrochage est l'abandon de l'apprentissage avant la fin de la formation ,sans passage de l'examen et sans signature d'un autre contrat. L'indicateur taux de rupture fait débat. Certains auteurs préfèrent calculer un taux de rupture net qui comptabilise les résiliations non suivies dans les trois mois par un autre contrat d'apprentissage, quand d'autres ne comptent pas soit les résiliations durant la période d'essai, soit les départs suite à l'obtention du diplôme.

L'approche méthodologique est donc longitudinale et comprend un suivi régulier des apprentis afin de mener une comparaison entre ces deux groupes. Des informations sur les apprentis de Côte-d'Or, leur scolarité, leurs acquis et les caractéristiques des entreprises ont été recueillies. Pour tester les capacités cognitives des apprentis, une sélection de tests du programme international pour le suivi des acquis des élèves (PISA) leur a été administrée en CFA (centre de formation d'apprentis). Les données quantitatives et qualitatives proviennent des missions locales, des chambres consulaires, des centres de formation, des questionnaires remplis par les apprentis dans les CFA, et d'une enquête téléphonique menée, en octobre 2011, auprès de 540 jeunes pour connaître leur situation et les raisons de leur départ de l'entreprise. Dès lors, la base de données spécifique à l'évaluation comprend des informations sur 1102 apprentis, 1223 entreprises de Côte-d'Or et plus de 80 variables. Par ailleurs, des entretiens semi-directifs ont été réalisés, en juin 2011, auprès des dix tutrices des missions locales de Côte-d'Or et de la coordinatrice du projet ; il s'agissait de connaître leurs points de vue, les aspects positifs et négatifs du tutorat et leurs suggestions pour l'améliorer.

Par la suite, ont aussi été mobilisés des observations (réunions, rencontres), des documents (contrats d'objectifs et de moyens, comptes rendus de réunions, rapports...), et de la documentation transmise par des acteurs ou provenant d'Internet (missions locales, conventions, blogs d'élus...)

\subsection{Un réseau d'acteurs}

Depuis 2002, la mission locale de Beaune proposait une action afin de réduire les taux d'abandon et de rupture dans l'hôtellerie-restauration. L'expérimentation a étendu ce dispositif à l'ensemble du département de la Côte-d'Or, en s'appuyant sur les pratiques menées avec l'Union des métiers et des industries de l'hôtellerie (Umih) de Côte-d'Or, l'association des restaurateurs du pays beaunois, la chambre de commerce et d'industrie de 
Beaune et le centre de formation d'apprentis de La Noue. Par ailleurs, un comité de pilotage a été créé dès le lancement du tutorat, composé de l'ensemble des acteurs ${ }^{7}$ régionaux et locaux impliqués dans la formation par alternance.

L'expérimentation a permis de nombreux rapprochements et la (re)connaissance des divers acteurs et institutions, habituellement cloisonnés, s'en trouve améliorée. La coordonnatrice du projet explique : "On ne peut pas aujourd'hui travailler tout seul autour de l'alternance et de l'apprentissage en particulier. " Des partenariats, des coopérations avec les centres de formation et les chambres consulaires ont été initiés afin de suivre au plus près les aléas des contrats d'apprentissage. Une dynamique partenariale et une synergie interinstitutionnelle ont été renforcées sur la zone de Beaune et développées sur deux autres territoires avec l'Umih. Les contacts et les collaborations avec les personnels des centres de formation sont fortement plébiscités par l'ensemble des tutrices ${ }^{8}$, avec un accueil positif et des retours d'informations complémentaires : "Très bonnes relations avec le CFA. Ils nous contactent mais c'est rare quand même, c'est plutôt dans l'autre sens que cela fonctionne. "Les partenariats avec les chambres consulaires leur paraissent plus compliqués, la circulation des informations, notamment la transmission des contrats d'apprentissage, étant parfois longue?. Quelquefois, les tutrices expriment une perception de concurrence ou de réticence de la part des personnels des chambres consulaires : "Quand j'ai eu des soucis, jai appelé plusieurs fois les chambres consulaires qui mont très bien répondu... mais elles mont plusieurs fois bien fait comprendre qu'elles étaient du côté des entreprises, que leurs clients, ce sont les entreprises."

Limportance du territoire apparaît à l'aune des acteurs mobilisés et des publics captés par les missions locales, jeunes et employeurs. Toutes les tutrices interrogées reconnaissent que cette mission leur a permis « de vraiment accompagner le jeune dans son parcours, être vraiment présente, plus proche des entreprises", de "partir à la rencontre des employeurs", "d'élargir les propositions de services vis-à-vis des employeurs", "de mettre en place un réseau entreprise ". Elles se positionnent comme une interface privilégiée entre les employeurs et les jeunes afin de proposer un regard objectif sur la situation, tout en confrontant les attentes des employeurs et les perceptions des jeunes quant au monde du travail.

Selon divers témoignages de tutrices, cet accompagnement est en général accepté par les jeunes qui ont le sentiment d'être écoutés : "cela les rassurait de savoir quills pouvaient compter sur quelquiun." Il est accepté aussi par la plupart des employeurs qui ont apprécié d'avoir une personne en externe pour optimiser la communication avec

\footnotetext{
7. Le service de l'apprentissage du rectorat, les centres de formation d'apprentis de Dijon et de Mercurey, les missions locales de Côte-d'Or (Beaune, Dijon, Montbard-Châtillon), les chambres de commerce et d'industrie de Beaune et de Dijon, la chambre régionale de métiers et de l'artisanat, l'Umih, le porteur du projet et l'équipe d'évaluation. Il était coprésidé par le Conseil régional (service de l'apprentissage) et la Direccte. 8. Le tutorat a été assuré par des conseillères des missions locales, ce qui explique le terme féminisé de tutrice. 9. La fusion institutionnelle des chambres de commerce de Beaune et de Dijon n'a pas facilité la transmission des contrats d'apprentissage et des résiliations.
} 
l'apprenti, même si l'accueil est différencié selon les secteurs. Ainsi, les petits domaines de la viticulture reflètent une ambiance plutôt familiale chez ces employeurs, qui connaissent la "génération Iphone dans les vignes ", respectueux vis-à-vis des apprentis et de la législation, avec l'envie de transmettre leur savoir-faire. Dans le commerce, à part quelques employeurs qui ne voyaient pas l'intérêt de cette action, les autres l'ont validée, «ils ont pris cela comme un appui, cela leur permettait de prendre du recul, de pouvoir discuter plus calmement"

En revanche, les tutrices relèvent davantage les contraintes et les particularités de l'hôtellerie-restauration où " tous les employeurs n'ont pas envie de jouer le jeu ". Une tutrice confirme : "On a noué de très bons contacts avec les entreprises, on est maintenant bien identifié. Là où c'est plus compliqué, c'est quand on intervient sur du conflit ", dans " un environnement professionnel très particulier, avec beaucoup de dérives. " C'est l'un des points négatifs relevés par les tutrices avec la difficulté de rester neutre face à des conflits et le manque de légitimité d'intervention par rapport à l'inspection du travail ou du CFA. Par exemple, lors de dépassements d'horaires ou d'abus en matière de conditions de travail, certaines insistent auprès des jeunes et des employeurs sur l'obligation de remplir des fiches horaires; elles fournissent aussi aux apprentis les contacts des services concernés comme l'inspection du Travail, ou sollicitent l'inspecteur de l'apprentissage, tout en maintenant une relative neutralité.

En fait, l'expérimentation a élargi le public habituel des missions locales, davantage d'apprentis dès le début de leur contrat, et a aussi créé une autre dynamique, dans le sens où les tutrices sont allées à la rencontre de ces jeunes et de leurs employeurs. Quels sont donc les impacts de cette action d'accompagnement sur les parcours des apprentis ? Peut-on remédier aux contraintes et aux difficultés des jeunes par des interactions rapides entre les employeurs et les missions locales?

\section{Tutorat et diminution des risques de rupture et d'abandon}

Dans le cadre de l'évaluation, 557 garçons et 545 filles apprentis de Côte-d'Or ont été suivis. Âgés en moyenne de 18 ans, ces jeunes sont plutôt d'origine modeste et composite : la moitié des nouveaux apprentis sont issus d'une classe de collège, le quart d'une section de lycée professionnel, $12 \%$ d'un lycée général et technologique, $3 \%$ d'une formation de l'enseignement supérieur. L'apprentissage reste une filière à prédominance masculine. Cependant, la répartition de la population est proche de la parité en raison de l'importance des apprenties de l'hôtellerie-restauration. La moitié des apprentis préparent un CAP, la part des jeunes en formation de niveau égal ou supérieur au baccalauréat est de $37 \%$. Les petites entreprises jouent un rôle essentiel : les trois quarts des jeunes se trouvent dans des établissements de moins de dix salariés. 
Lancienneté professionnelle des maîtres d'apprentissage est importante, $40 \%$ travaillent depuis plus de vingt ans, et la part des non-diplômés est conséquente (46\%).

Parmi les 1102 jeunes suivis, 532 ont bénéficié du tutorat, 377 ont été informés par courrier et 193 ne sont pas entrés dans l'expérimentation (tableau 1). Les ruptures sont déterminées au regard du contrat de travail, lorsque la date prévisionnelle de fin de contrat n'est pas atteinte sur la période observée, soit d'octobre 2009 à octobre 2011. Au cours de ces deux années, 508 apprentis ont connu au moins une résiliation, dont 202 étaient en tutorat et 165 informés par lettre. Toutes les entreprises sont confrontées à ce problème, plus souvent dans l'hôtellerie-restauration et le commerce, qui sont des secteurs à fort taux de rotation de la main-d'œuvre. Pour autant, la résiliation ne signifie pas toujours l'échec - comme le cas positif d'une embauche directe sur un poste stable - et n'indique pas forcément l'abandon de l'apprentissage ou du métier puisque nombreux sont les jeunes qui signent un nouveau contrat. Au final, 167 jeunes (303 avec les non-bénéficiaires) ont quitté définitivement l'apprentissage.

Tableau 1. Caractéristiques des apprentis et nombre de ruptures

\begin{tabular}{|lcc|}
\hline & Apprentis suivis & Ruptures de contrats \\
\hline Jeunes en tutorat & 532 & 202 \\
\hline $\begin{array}{l}\text { Jeunes informés par lettre de } \\
\text { l'existence du tutorat }\end{array}$ & 377 & 165 \\
\hline $\begin{array}{l}\text { Jeunes non-bénéficiaires (ni lettre ni } \\
\text { proposition de tutorat) }\end{array}$ & 193 & 141 \\
\hline Hôtellerie-restauration & 711 & 382 \\
\hline Commerce & 261 & 112 \\
\hline Viticulture & 130 & 14 \\
\hline $\begin{array}{l}\text { Effectifs entreprise } \\
\text { 0à 4 salariés }\end{array}$ 5à 9 salariés & 504 & 217 \\
\hline Garçons & 318 & 153 \\
\hline Filles & 557 & 245 \\
\hline Ensemble & 545 & 263 \\
\hline
\end{tabular}

Champ : ensemble des apprentis suivis (1 102 jeunes).

Lecture : parmi les 532 bénéficiaires du tutorat, 202 ont connu une rupture de contrat.

Source : enquête IREDU. 
Dans un premier temps, les effets du tutorat et de la lettre d'information ont été évalués par un modèle logistique ${ }^{10}$ : rupture ou non, abandon de l'apprentissage ou non. Le tutorat, mené par les missions locales auprès des apprentis et des employeurs, suggère une réelle plus-value ; en effet, il diminue, de manière significative, le nombre de ruptures de contrat et d'abandons de l'apprentissage.

À caractéristiques sociales, scolaires et d'entreprises équivalentes, le jeune informé par lettre a plus de risques de connaitre une rupture du contrat (multiplié par 1,6) et un abandon de l'apprentissage (multiplié par 2,3) qu’un jeune bénéficiaire du tutorat (tableau 2). Les principaux facteurs qui influencent le départ de l'entreprise sont l'âge de l'apprenti - les plus jeunes abandonnent davantage -, sa relation avec le maître d'apprentissage, l'activité de l'entreprise. La qualité de la relation avec le maître d'apprentissage est un enjeu important qui peut entrainer un départ en cas d'insatisfaction. Par rapport aux apprentis de la viticulture, ceux du commerce et surtout ceux de l'hôtellerie-restauration ont plus de risques de connaître une résiliation de contrat. Aucune différence significative n’apparaît concernant le sexe de l'apprenti ni son origine sociale ${ }^{11}$.

Dans un second temps, le protocole d'assignation aléatoire permet d'obtenir une mesure de ce qu'apporte l'attribution d'emblée d'un tuteur par rapport à l'envoi d'une lettre d'information, grâce à l'approche de la double différence ${ }^{12}$. Cette méthode est exclusivement fondée sur l'analyse des variables de résultat (rupture, maintien), en éliminant les effets fixes (variables de secteur, âge, sexe, origine sociale et scolaire, bilan des acquis), puis temporels, à l'aide de deux différences successives. Il s'agit de comparer les changements au fil du temps (la première différence concernant les situations en début et fin d'expérimentation) sur les deux populations (la seconde différence). La différence entre ces deux mesures permet ainsi d'isoler l'effet du dispositif. Le résultat le plus significatif apparaît pour les apprentis préparant un bac pro de l'hôtellerie-restauration (tableau 3). Le tutorat conduit à une minoration du risque de rupture d'environ huit points par rapport aux apprentis informés par lettre préparant le même diplôme, toutes choses égales par ailleurs. Pour les jeunes en CAP et BEP du commerce et de

10. La régression logistique " toutes choses égales par ailleurs " a été privilégiée afin d'isoler les effets spécifiques du tutorat, des caractéristiques individuelles de l'apprenti (genre, âge, origine sociale et scolaire, diplôme préparé, mode de logement, choix du métier, bilan des acquis...), de celles du contexte de l'apprentissage (localisation de l'entreprise, diplôme du maître d'apprentissage, ancienneté...).

11. La modélisation effectuée sur l'ensemble des 1102 apprentis souligne aussi l'impact du tutorat sur les ruptures et les abandons. En revanche, le tutorat n'a aucun effet sur l'accès à l'emploi des jeunes. Ce n'était pas l'objectif premier du projet qui devait sécuriser le parcours des apprentis dès la signature du contrat. De plus, l'insertion professionnelle étant un processus qui s'inscrit dans la durée, un délai de plusieurs années est nécessaire pour obtenir une vision significative de l'entrée des jeunes dans la vie active. Par ailleurs, cela ne concerne qu'un faible effectif d'actifs qui n’ont pas poursuivi leurs études (197 jeunes). Ces résultats ont été présentés aux acteurs et sont mentionnés dans le rapport final d'évaluation (Bourdon, Guégnard, Michot, 2012).

12. Pour une présentation des méthodes d'évaluation, $c f$. Brodaty, Crépon, Fougère (2007) et Givord (2010). 
l'hôtellerie-restauration, la diminution du risque de rupture est de l'ordre de six points et l'essentiel des effets peut être imputé au dispositif ${ }^{13}$.

Tableau 2. Impacts du tutorat sur la rupture du contrat et l'abandon de la formation

\begin{tabular}{|c|c|c|c|}
\hline & & $\begin{array}{l}\text { Rupture } \\
\text { du contrat }\end{array}$ & $\begin{array}{c}\text { Abandon } \\
\text { de } \\
\text { I'apprentissage }\end{array}$ \\
\hline Tutorat & Information & 1.6 & 2.3 \\
\hline Secteur & Hôtellerie-restauration & 9.1 & $18.2^{*}$ \\
\hline Viticulture & Commerce & $4.5^{*}$ & $9.5^{*}$ \\
\hline Âge & & $0.9^{*}$ & $0.9^{*}$ \\
\hline $\begin{array}{l}\text { Origine scolaire avant CFA } \\
\text { Collège }\end{array}$ & Lycée général & 2.8 & $1.9^{*}$ \\
\hline $\begin{array}{l}\text { Satisfait du maître } \\
\text { d'apprentissage }\end{array}$ & Insatisfait & $2.4^{*}$ & $2.8^{*}$ \\
\hline $\begin{array}{l}\text { Localisation entreprise } \\
\text { Zone de Beaune }\end{array}$ & $\begin{array}{l}\text { Dijon } \\
\text { Montbard } \\
\text { Hors Côte-d'Or }\end{array}$ & $\begin{array}{c}\text { ns } \\
\mathbf{0 , 4 ^ { * }} \\
\text { ns }\end{array}$ & $\begin{array}{l}\text { ns } \\
\text { ns } \\
\text { ns }\end{array}$ \\
\hline $\begin{array}{l}\text { Effectifs } \\
\text { Nombre de ruptures/abandons }\end{array}$ & & $\begin{array}{l}723 \\
278\end{array}$ & $\begin{array}{l}723 \\
167\end{array}$ \\
\hline Pseudo R-deux & & 0.30 & 0.33 \\
\hline
\end{tabular}

Les résultats sont présentés en odds ratio (rapports de chances) et sont significatifs (chiffres en gras) au seuil de $1 \%$ (10\% s'ils sont signalés par une *), la mention «ns » indiquant un effet non significatif. Certaines variables explicatives non significatives ne sont pas présentées (diplôme préparé, genre, origine sociale, mode de logement, choix du métier, bilan des acquis, localisation et effectifs de l'entreprise, caractéristiques du maitre d'apprentissage...). Champ : bénéficiaires du tutorat et de la lettre d'information (723 jeunes).

CFA : centre de formation d'apprentis.

Lecture : toutes choses égales par ailleurs, le jeune informé par lettre a 1,6 fois plus de risques de rupture du contrat et 2 fois plus de risques de quitter le secteur qu'un jeune bénéficiaire du tutorat (significatifs au seuil de $1 \%$ ).

Source : enquête IREDU.

Tableau 3. Effets du tutorat sur le risque de rupture par rapport à la lettre d'information

\begin{tabular}{|lcc|}
\hline & Bac Pro & CAP-BEP \\
\hline Hôtellerie-restauration & 8,6 & $-6,3^{*}$ \\
\hline Commerce & ns & $-6,2^{*}$ \\
\hline Ensemble & $7,6^{*}$ & $-6,2^{*}$ \\
\hline
\end{tabular}

Champ : bénéficiaires du tutorat et de la lettre d'information (723 jeunes).

Les chiffres sont significatifs au seuil de $1 \%$ (10\% s'ils sont signalés par une *), la mention «ns » indiquant un effet non significatif.

Lecture : pour les apprentis de l'hôtellerie-restauration préparant un bac pro, le tutorat conduit à une minoration relative du risque de rupture de près de 8 points par rapport aux apprentis informés par lettre préparant le même diplôme (significatif au seuil de $1 \%$ ).

CAP-BEP : certificat d'aptitude professionnelle ; brevet d'études professionnelles.

Source : enquête IREDU.

13. Ce constat d'un risque de rupture supérieur pour les contrats les plus longs s'accorde avec les conclusions de la littérature (Malcomson et al., 2003). 
Au vu de ces résultats, l'IREDU s'est prononcé sur la vocation du tutorat à être répliqué dans d'autres territoires ${ }^{14}$. À la demande des acteurs régionaux, les principaux enseignements de cette évaluation et les hypothèses, quant aux idées ou pratiques transférables vers d'autres zones géographiques, ont été exposés. Par la suite, en référence au contrat d'objectifs et de moyens, une décision politique a été prise. Elle sera présentée dans la partie suivante, au regard des stratégies des principaux acteurs.

\section{De l'avis de transférabilité à la décision politique}

L'IREDU a participé à deux réunions du Comité régional pour l'insertion des jeunes (Corij), instance de pilotage de l'animation de la politique d'insertion des jeunes au sein des missions locales, qui réunit des acteurs régionaux et locaux ${ }^{15}$. L'objet de ces réunions portait sur l'extension de l'expérimentation du tutorat en Bourgogne, basée sur son évaluation et la présentation des divers dispositifs d'accompagnement mis en place par les consulaires (développeurs de l'apprentissage, médiateurs de la chambre de métiers...), et par le conseil régional (aides versées aux employeurs et aux apprentis, formation des maîtres d'apprentissage...).

La participation à ces rencontres a permis d'approcher le positionnement pluriel et complexe des acteurs et de leurs stratégies dans le domaine de l'apprentissage. Ce comité paraît constituer un lieu favorable à la réduction de l'incomplétude de l'information et à l'appropriation de l'information par les acteurs ; éléments qui paraissent nécessaires avant toute transaction, afin d'adopter une représentation commune en termes d'accompagnement des apprentis.

\subsection{L'évaluateur propose l'extension du dispositif}

Lors de ces réunions, l'IREDU a présenté les perspectives de transfert du dispositif. Répliquer le tutorat à une échelle similaire paraît réalisable dans la mesure où il a déjà été développé par deux autres missions locales et sur deux autres secteurs. Il est primordial d'accompagner les apprentis dès leurs premiers pas dans le monde de l'entreprise jusqu'à la fin du contrat, au vu des ruptures au-delà de la période d'essai des deux mois. La prio-

14. $C f$. Rapport d'évaluation sur le site www.experimentation.jeunes.gouv.fr.

15. Le Corij a été mis en place par une convention signée en septembre 2000, entre l'Association régionale des missions locales de Bourgogne, le conseil régional et l'État. Il se compose de : cinq représentants de l'État (Secrétaire général aux affaires régionales, Direccte, Droit des Femmes, Éducation nationale, Pôle Emploi), cinq représentants du conseil régional, cinq présidents de missions locales, conseils généraux. Ses objectifs sont d'impulser des politiques d'insertion menées en direction des jeunes, de définir des objectifs et priorités partagés, de contribuer à la définition d'une politique de formation professionnelle, de procéder à une analyse qualitative des dispositifs et actions portés ou conduits par les missions locales (extraits de la convention). 
rité portera davantage sur les apprentis de première année de CAP (certificat d'aptitude professionnelle) et Bac Pro. À cette fin, l'échange d'informations entre les centres de formation et les missions locales concernant les jeunes doit être constant dans le temps; de même, les relations avec les chambres consulaires doivent perdurer, particulièrement au moment des enregistrements et résiliations de contrats, mais aussi lors de la recherche d'entreprises. L'essaimage peut s'effectuer en ne prenant qu'un seul secteur, comme l'hôtellerie-restauration pour toutes les missions locales de Bourgogne, avec une coordination régionale. L'alternative est de mettre l'accent sur le département de Côte-d'Or avec la continuité sur les trois secteurs initiaux pour conserver la synergie des acteurs, et une extension à d'autres secteurs. Par la suite, cet accompagnement peut être disséminé à d'autres territoires volontaires et régions, au vu des résultats. Ce dispositif porté par les missions locales devra bénéficier d'un soutien financier et d'une formation des tuteurs en s'appuyant sur les expériences acquises. La qualité de l'accueil et de la formation des jeunes en entreprise représentant un enjeu important, des pistes complémentaires sont à envisager, comme une charte des entreprises, un engagement entre l'apprenti et le maître d'apprentissage, des primes versées aux entreprises avec obligation de résultats, une réelle formation des maitres d'apprentissage... et la création d'un observatoire de l'apprentissage au plan régional, voire national. Il pourrait analyser les ruptures, mener des enquêtes auprès des apprentis et des employeurs, réaliser des évaluations tant sur le plan quantitatif que qualitatif, tel un observatoire de la vie des apprentis.

Dans l'ensemble, les propositions de l'IREDU rejoignent celles exprimées par le porteur du projet ${ }^{16}$ qui insiste davantage sur le soutien de l'État et de la Région pour légitimer l'action, tant en termes de financement que de pilotage du dispositif, avec des indicateurs d'évaluation, une communication, et le choix d'un tuteur pour 150 apprentis. Le directeur de la mission locale insiste sur le suivi des jeunes placés en alternance par les missions locales jusqu'à la fin des contrats plus trois mois. Il s'agit de pouvoir intervenir rapidement auprès des apprentis en situation de rupture sans attendre plusieurs mois de décrochage et d'errance. Il incite à ne prendre qu'un seul secteur emblématique, comme l'hôtellerierestauration, pour toutes les missions locales de Bourgogne, et il précise que l'Umih est favorable à l'extension.

\subsection{De l'intermédiation à l'interdépendance...}

Suite à la présentation des perspectives d'extension du tutorat en Bourgogne, les débats ont été marqués par quatre principaux acteurs, avec des représentations et des résistances autour de l'enjeu de la sécurisation des parcours des apprentis.

16. $C f$. Note de restitution finale du porteur du projet sur le site www.experimentation.jeunes.gouv.fr. 
Un nouvel acteur ou plutôt un groupement d'acteurs est entré en jeu. En effet, l'Association régionale des missions locales de Bourgogne (Assor) ${ }^{17}$ permet au réseau des missions locales d'assurer "un rôle d'interface régulier avec les grands partenaires régionaux (État, Région) et d'appui à la mise en ouvre de leurs programmes : formation des jeunes, orientation, accompagnement, mobilité, lutte contre le décrochage scolaire, accompagnement vers l'emploi, l'alternance ». La position du président de l'Assor ${ }^{18}$ a fortement évolué au cours de ces rencontres. Lors de la première réunion centrée sur la généralisation du tutorat, il s'exprime ainsi : "Le choix sera à faire entre tous les métiers de Côte-d'Or, ou un métier/une branche sur toute la Bourgogne, la seconde solution étant plus intéressante en ciblant sur l'hôtellerie-restauration, ce qui serait cohérent avec le projet de développer le tourisme en Bourgogne. "À l'issue de la seconde réunion, sa posture change puisqu'il estime qu'il ne faut pas surajouter des dispositifs et qu'il convient de mener un travail technique pour identifier les secteurs et les périmètres, travail qui pourra servir de base à une convention. Il conclut en proposant une poursuite des travaux par une réunion restreinte et technique entre l'Assor, le conseil régional et la Direccte, par l'envoi d'une liste de commande aux différents acteurs, puis une réunion du Corij pour la synthèse. En parallèle, il propose la poursuite de la réflexion propre au secteur de l'hôtellerie-restauration dans le cadre du contrat d'objectifs territoriaux (COT).

Deuxième groupe d'acteurs, les chambres consulaires se positionnent au service des artisans et des entreprises et présentent leurs actions concernant l'apprentissage, qui est une de leur "mission régalienne », selon leurs propres termes. Cogestionnaires des CFA, elles favorisent l'apprentissage en renseignant sur la réglementation, les métiers et les filières de formation, en recensant l'offre et la demande, en établissant les contrats, en accompagnant le maître d'apprentissage par des formations, en proposant une médiation en cas de difficulté. À cet égard, des tensions sont apparues autour des interventions en entreprise et de la notion de médiation. Cette fonction est assurée par la chambre de métiers et de l'artisanat qui indique que son médiateur mis en place depuis six ans est de plus en plus sollicité, et qui insiste sur sa connaissance des entreprises. Les représentants des consulaires formulent des interrogations quant à la connaissance des entreprises par les missions locales, suggérant qu'elles ne devraient s'adresser qu'aux jeunes, les consulaires conservant les relations avec les entreprises. De plus, ils soulignent que la généralisation du tutorat générerait des engagements financiers.

En écho, la mission locale de Beaune précise qu'il faut différencier et non concurrencer le tutorat de la médiation. En effet, la médiation est une intervention ponctuelle, un processus amiable de résolution de conflit. Le tutorat quant à lui consiste en un accompagnement pour prévenir et répondre aux difficultés que peut rencontrer un jeune tant

17. Créée il y a 15 ans, $C f$. site www.missions-locales-bourgogne.fr.

18. Le président de l'Assor est aussi président du Corij, d'une mission locale impliquée dans l'expérimentation et conseiller régional, ce qui complexifie son rôle d'interface. 
au niveau professionnel (entreprise, CFA) que social (logement, mobilité, finances...). Il se met en place à la signature du contrat et perdure jusqu'à son terme pour des secteurs présentant une certaine instabilité.

Du côté de l'État, le représentant de l'inspection de l'apprentissage défend la place centrale des CFA définie par le Code du travail. Il insiste sur la nécessité d'analyser les causes des ruptures, en positionnant les CFA au cour de la problématique, et sur le renforcement de la formation des maîtres d'apprentissage. À son avis, l'apprentissage se fonde sur le trio CFA/entreprise/apprenti. Il cite notamment l'article (R6233-58) qui prévoit, dans les deux mois suivant la conclusion du contrat, un entretien d'évaluation mené par le CFA avec l'apprenti, l'employeur, le maître d'apprentissage. L'enjeu essentiel serait de faire intervenir un partenaire extérieur, comme la mission locale, en confortant le rôle des CFA qui ont en charge de faire vivre l'alternance.

La Région, pour sa part, se trouve en position d'intermédiaire entre le monde des entreprises et celui de l'éducation, tout en assurant la gestion des dispositifs de formation constitués par l'apprentissage, la formation professionnelle des jeunes et des adultes. L'apport du conseil régional est de préciser à l'ensemble des partenaires la nature des enjeux, de recenser et défendre les dispositifs d'actions présents. Il rappelle le budget conséquent alloué à l'apprentissage et les mesures de suivi des apprentis, qui sont confiées aux CFA et font l'objet de financements particuliers (dont les visites d'entreprises réalisées avant le 31 décembre, le contrôle en cours de formation...), les aides versées aux entreprises en fonction de l'assiduité au CFA. Il insiste sur le COT de l'hôtellerie-restauration qui intègre une réflexion sur l'accueil des apprentis, et sur l'objectif de la branche, au plan national, de rendre obligatoire la formation des maîtres d'apprentissage. Le conseil régional est un acteur pivot mais est aussi partie prenante au titre des financements qu'il assure.

Les discussions lors de ces réunions ont aussi porté sur les relations entre missions locales, consulaires et CFA, la démarche à adapter aux besoins différenciés des branches professionnelles et des territoires. Au fil des échanges, il s'est avéré que les contenus et l'intensité des coopérations variaient selon les territoires, les collaborations des missions locales pouvant être localement plus ou moins faciles avec les consulaires. Au final, la construction d'une représentation commune de l'intervention auprès des apprentis et des entreprises n'a pas eu lieu. Dès lors qu'une définition collective du concept de rupture et des motifs n'a pu être élaborée, il est difficile d'aboutir à une vision convergente des modes d'intervention auprès des entreprises et des jeunes pour y remédier. En effet, la rupture ne recouvre ni le même sens, ni le même chiffre selon les institutions présentes : avec ou sans les deux mois d'essai, rupture de contrat avec un changement d'entreprise, départ définitif du centre de formation...

Chaque acteur défend son institution et ses dispositifs, qui n’ont pourtant pas bénéficié d'évaluation externe, plutôt que d'investir humainement et financièrement dans le tutorat, action ayant un impact sur la rupture ou le décrochage des apprentis. 
Ces diverses positions témoignent des difficultés de compréhension qui se multiplient à l'occasion des arbitrages sur les programmes de formation et d'actions, dans l'exercice d'une compétence partagée "dans un univers complexe de gouvernance éclatée où l'État et les régions ne sont que des partenaires parmi d'autres" (Arrighi, 2011).

Se retrouvent ici les principales sources des coûts de transaction. D'une part, par l'incompatibilité de but qui caractérise une situation où l'une des parties a l'intention de promouvoir ses propres intérêts au détriment de l'autre. D’autre part, par l'ambiguïté de la performance résultant de l'impossibilité de mesurer la performance des parties dans un échange, ou d'une incapacité à la valoriser correctement, même si la performance est évaluée, comme ici le tutorat.

\section{3... à la gouvernance}

Le Corij, cadre d'action collective à un réseau d'acteurs, offre une scène originale de négociation ou de dialogue social, dont la coordination oscille entre concurrence et coopération (Mériaux, Verdier, 2009). Le processus décisionnel paraît complexe, avec des temps de discussion et de négociation entre acteurs aux stratégies différenciées et porteurs d'informations spécifiques. La décision finale du conseil régional a été de continuer à privilégier le financement de la visite des apprentis en première année par le personnel enseignant des CFA au cours des premiers mois du contrat d'apprentissage ${ }^{19}$. Pourtant, dans le contrat d'objectifs et de moyens pour l'apprentissage sont inscrites, parmi les actions à conduire: " des mesures à caractère pédagogique (personnalisation des parcours, visites en entreprises, médiation, tutorat externe...) incontournables pour préserver les taux de rupture de contrat (...). Par expérience, ces mesures sont essentielles pour diminuer les taux de rupture et maintenir un haut niveau de qualité. " La proximité des organismes mobilisés sur le terrain a fait pencher la balance du côté des CFA, du fait de liens particuliers entre CFA et conseil régional. En effet, la Région signe des conventions avec les CFA, leur attribue des financements de fonctionnement et d'investissement, planifie l'ouverture et la fermeture des formations par apprentissage. L'existence d'une relation ni anonyme ni limitée dans le temps a créé une dépendance qui a modifié la règle contractuelle habituelle.

Cette décision peut aussi être perçue comme une minimisation des coûts de transaction à travers le maintien d'une relative paix politique. Le choix d'une extension du tutorat aux missions locales de la région aurait nécessité d'autres coûts plus élevés (embauche, formation, coordination), et des coûts associés à la négociation, la rédaction, la gestion..., et ce dans un avenir incertain. En privilégiant le mode d'organisation fondé sur l'implication des CFA, le conseil régional s'assure ainsi de la continuité d'une pratique actuelle et d'une qualité attendue des prestations prévues par la loi et soutenues par un financement. Lors de la mise en œuvre de ces transactions, des agents peuvent

19. $150 €$ par visite d'un jeune en entreprise. La mission locale proposait $300 €$ pour l'accompagnement de 150 apprentis pendant toute l'année et non sur une seule visite. 
avoir un rôle plus ou moins influent dans un contexte de divergence d'appréciations, comme l'inspecteur de l'apprentissage qui argumentait afin de placer les CFA au cœur du dispositif, en référence à la législation, alors que les chambres consulaires s'opposaient à l'extension du tutorat.

Enfin, les conditions du déroulement postérieur d'une relation contractuelle peuvent jouer un rôle fondamental. Effectivement, la mise en œuvre du contrat peut être perturbée dans certains cas. Il s'agit du comportement dit "opportuniste " selon Williamson, où un acteur agit dans son propre interêt parce qu'il existe un désaccord dû à une divergence d'appréciation d'une situation. Suite au renoncement de l'extension du tutorat, la mission locale de Beaune a obtenu une subvention de l'État, ce qui lui a permis de poursuivre son action de tutorat auprès des jeunes et des employeurs quelques mois supplémentaires.

\section{Conclusion}

Au final, cette étude de cas amène plusieurs remarques. En premier lieu, il est indispensable de distinguer la notion de gouvernance de celle de gouverner. La gouvernance définie par Bagnasco et Le Galès (1997) est un processus de coordination d'acteurs, de groupes sociaux, d'institutions pour atteindre des buts propres discutés et définis collectivement dans des environnements fragmentés et incertains. Elle suppose de préciser auparavant, ou de façon concomitante à l'action, la nature des relations contractuelles. Ces dernières sont élaborées par des acteurs dont l'analyse des stratégies devient déterminante.

Le deuxième commentaire renvoie à l'enjeu que représente l'information sur l'apprentissage dans l'élaboration de ces relations contractuelles. Le cadre théorique général de la rationalité limitée, dans laquelle s'inscrit la théorie des coûts de transaction, considère que l'information disponible pour qu'un agent puisse prendre une décision est incomplète, souvent fragmentaire, et qu'il est difficile de connaître les conséquences des différentes possibilités d'action. La nature de l'information, l'étendue de sa diffusion et ses modalités d'appropriation, par les acteurs, constituent un des enjeux de réussite du modèle de la gouvernance. À cet égard, les rôles du Corij (Comité régional pour l'insertion des jeunes) et du conseil régional seront d'autant plus efficaces qu'ils contribueront à réduire l'incomplétude de l'information dans le cadre d'une gouvernance complexe à laquelle participent de multiples partenaires.

L'impact positif d'un tutorat en direction des jeunes et des employeurs, mené par les missions locales, est ici mis en lumière pour assurer la continuité des parcours en apprentissage. D'autres dispositifs concernant les apprentis, et financés par le Fonds d'expérimentation pour la jeunesse, ont été réalisés ou sont en cours d'évaluation, mais ils diffèrent par les publics visés, les actions des missions locales, les méthodologies mises en œuvre. 
Dans un $\operatorname{cas}^{20}$, les résultats montrent que l'accompagnement en amont de la signature du contrat d'apprentissage ne permet pas de diminuer le nombre des ruptures.

Face à une validation scientifique, l'histoire de cette expérimentation renvoie aussi à l'impact limité de l'expertise au regard des décisions politiques, d'autant plus que la combinaison entre logiques d'acteurs est forte. Jusqu'à quel point les acteurs, dans une conjoncture économique difficile, sont-ils prêts à prendre en compte les conclusions d'une évaluation externe qu'ils ont eux-mêmes en partie financée ? Ceci révèle " la fragilité d'une conception du recours à l'expertise comme technique de rationalisation de l'action publique ", soulignée par Freyssinet (2008, p. 210). Le passage d'une expérimentation, même réussie, à une extension, ne peut se limiter à la seule transposition des résultats de l'évaluation et montre l'importance des coordinations et des transactions entre acteurs institutionnels. L'analyse des choix politiques, dans le domaine de la relation formation-emploi, réalisés par une Région dans un univers complexe d'interdépendance est captivante... et continuera de l'être lors de l'acte III de la décentralisation.

\section{- Bibliographie}

Abriac D., Rathelot R., Sanchez R. (2009), "L'apprentissage entre formation et insertion professionnelles ", in Insee, Formations et Emploi, pp. 57-74.

Alet E., Bonnal L. (2013), «L'apprentissage : un impact positif sur la réussite scolaire des niveaux V", Économie et Statistiques, 454, pp. 3-22.

Arrighi J.-J. (2011), "Formations en apprentissage : en finir avec les illusions ", Synthèse Terra Nova, La Fondation progressiste, 11 p.

Bagnasco A., Le Galès P. (dir.) (1997), Villes en Europe, Paris, La Découverte.

Bentabet E., Cart B., Henguelle V., Toutin M.-H. (2012), « Jeunes et entreprises face aux ruptures de contrat d'apprentissage ", Commissions professionnelles consultatives études, 1, $245 \mathrm{p}$.

Bourdon J., Guégnard C., Michot C. (2012), Inscrire les contrats en alternance dans une logique de parcours sécurisé, Rapport d'évaluation IREDU/CNRS, Fonds d'expérimentation pour la Jeunesse, ministère de l'Éducation nationale, de la Jeunesse et de la Vie associative, $149 \mathrm{p}$.

Bourdon J., Guégnard C., Michot C. (2012), "Sécuriser les parcours des apprentis », Bref-Céreq, 301. 
Brodaty T., Crépon B., Fougère D. (2007), « Les méthodes micro-économétriques d'évaluation et leurs applications aux politiques actives de l'emploi ", Économie et Prévision, 1(177), pp. 91-118.

Cart B., Ducourant H., Henguelle V., Surelle A.-S. et Toutin M.-H. (2007), Les ruptures de contrats d'apprentissage en région Nord-Pas de Calais, Rapport Car-Céreq Lille, 162 p.

Céreq (2011), "Le diplôme : un atout gagnant pour les jeunes face à la crise ", Bref-Céreq, 283.

Chambres de commerce et d'industrie (2010), Les ruptures de contrats d'apprentissage... une fatalité ? Synthèse, $4 \mathrm{p}$.

Comité de coordination des programmes régionaux d'apprentissage et de formation professionnelle continue (2000), Evaluation des politiques régionales de formation professionnelle 1997-1999, Vol. 1, Rapports, La Documentation française, Paris.

CREST et DARES (2012), Sécurisation du parcours des jeunes s'engageant dans l'apprentissage : bilan d'une expérimentation aléatoire contrôlée conduite en Corrèze, Rapport d'évaluation, Fonds d'expérimentation pour la jeunesse, ministère de l'Éducation nationale, de la Jeunesse et de la Vie associative, 88 p.

Direction de l'évaluation et de la prospective, (1995), "Apprentissage : les ruptures de contrat vues par les jeunes et les employeurs ", Note d'information, 95.38, ministère de l'Éducation nationale, septembre.

Direction de l'évaluation et de la prospective, (1997), "Apprentissage : ruptures, enchaînements de contrats et accès à l'emploi ", Note d'information, 97.22, ministère de l'Éducation nationale, avril.

Freyssinet J. (2008), «L'expert entre le savant et le politique : l'efficacité des rapports d'expertise sur l'emploi et la formation ", Formation Emploi, 101, pp. 199-212.

Giffard A., Guégnard C. (2003), «Territoire, formation, gouvernance : des relations complexes et paradoxales ", Formation Emploi, 84, pp. 55-65.

Givord P. (2010), "Méthodes économétriques pour l'évaluation des politiques publiques », Document de travail, Insee. [En ligne] http:/www.insee.fr/fr/publications-et-services/ docs_doc_travail/G2010-08.pdf (consultée le 15 février 2011).

GREF (2013), Les ruptures d'apprentissage en Bretagne, Mission Observatoire emploi-formation, $28 \mathrm{p}$.

Malcomson J.M., McGaw J.W., McCormick B. (2003), "General training by firms, apprentice contracts, and public policy”, European Economic Review, 47, pp. 197-227.

Mériaux O., Verdier E. (2009), «Compétences territoriales et émergence d'une politique du rapport salarial », Espaces et Sociétés, 136-137, pp. 17-31. 
Moreau G. (2003), Le monde apprenti, Paris, La Dispute.

Moreau G. (2008), "Apprentissage : une singulière métamorphose », Formation Emploi, 101, pp. 119-133.

Sollogoub M., Ulrich V. (1999), «Les jeunes en apprentissage ou en lycée professionnel : Une mesure quantitative et qualitative de leur insertion sur le marché du travail ", Économie et Statistiques, 323, pp. 31-52.

Steedman H. (2012), Overview of Apprenticeship Systems and Issues, ILO contribution to the G20 Task Force on Employment, September, BIT, Genève, [En ligne]: http://www. ilo.org/wcmsp5/groups/public/ed_emp/ifp_skills/documents/genericdocument/wcms_190188. pdf (consulté le 2 octobre 2013).

Williamson O.E. (1985), The Economic Institutions of Capitalism. Firms, Markets, Relational contracting, New York, The Free Press, Coll. Mac Millan Publisher. Traduit en français (1994) Les institutions de l'économie, InterEdition Paris. 